\title{
Nanomedicine and Immunotherapy for Cancers
}

\author{
A. A. Navas, N. Doreswamy, and P. J. Joseph Francis
}

\section{ABSTRACT}

\begin{abstract}
Nanomedicine contributes to cancer therapeutics in several ways, harnessing some of the remarkable properties of nanomaterials to target tumor cells with increasing specificity. Nano-scale therapeutic strategies enable the simultaneous transport of hydrophilic and hydrophobic drugs across physical and physiological barriers like the blood-brain barrier. Alternative routes of drug administration, such as the intranasal route, have become viable, with more promising therapies for highly lethal tumors like glioblastomas. Cancer nanomedicine allows increased solubility and bioavailability of anti-cancer drugs, reducing their toxicity.

Multi-drug delivery systems such as dendrimers, noble metal nanoparticle drug delivery systems, nanoparticle-based theranostics, and nanobiomarkers may well bring about a sea change in cancer therapeutics. This review presents an overview of the scope of cancer nanomedicine, including immunotherapy for cancer.
\end{abstract}

Keywords: Nanomedicine, Immunotherapy, Theranostics, Tumor heterogeneity, Cytokine Release Syndrome, Cancer Nanomedicine.
Published Online: September 16, 2020

ISSN: 2593-8339

DOI: $10.24018 /$ ejmed.2020.2.5.482

\section{A. A. Navas}

Department of Internal Medicine, College of Medicine and Health Sciences, National University of Science and Technology, Sultanate of Oman. (e-mail: navaskj@ nu.edu.om)

N. Doreswamy

National Coalition of Independent Scholars, Australia.

(e-mail: ndoreswamy@outlook.com) P. J. Joseph Francis*

Department of Biochemistry, College of Medicine and Health Sciences, National University of Science and Technology, Sultanate of Oman.

(e-mail: josephfrancis ${ }^{\circledR}$ nu.edu.om)

*Corresponding Author

\section{INTRODUCTION}

Nanomedicine, the emerging miracle of the last decades, is finally at the threshold of broader clinical acceptance and approval. Cancer nanomedicine may be the game changer that achieves a long-standing therapeutic objective for all malignancies: safe treatment protocols with low toxicity, and robust, complete, curative end points in the patient's journey back to health and quality of life.

We are at the helm of a new era of highly selective cancer treatments that are able to utilize engineered and targeted strategies at the nano scale. Of particular interest is the promise of boosting the patient's own immunity, facilitating a more robust immune memory, preventing recurrence and improving long-term survival.

Here, we undertake a review of nanomedicine in the treatment of cancers, with a focus on tumor heterogeneity, immunotherapy, and the potential for potent combination therapies in conjunction with conventional treatments such as chemotherapy and radiotherapy.

\section{CANCER NANOMEDICINE}

Nanomedicine uses nanomaterials for the prevention, control, diagnosis, monitoring, and treatment of diseases [1]. Cancer nanomedicine applies nanomedicine to cancers. Conventional cancer therapies are widely used in clinical practice, but have several limitations - inadequate tissue selectivity, drug resistance over time, and poor tissue concentration reduce their efficacy. Cancer nanomedicine has the potential to overcome these limitations [2].

In general, nanomedicine harnesses the remarkable properties of nanoparticles (NPs) - which, by definition, are 1 to 100 nanometers $(\mathrm{nm})$ in size in at least one dimension [3]. The smallest dimension of an NP can be just twice the size of a large atom like Cesium [4]. NPs are about the same size as viruses and small bacteria; this may be the reason why NPs stimulate the immune system [5]. A size of less than $100 \mathrm{~nm}$ also enables them to take advantage of the increased permeability and retention seen in solid tumors [6].

Drug dosage form design, the absorption and efficacy of drugs administered by the oral route, have been of concern and interest for many decades [7]. When administered as nanocrystals less than $1 \mu \mathrm{m}$, drugs show significantly higher oral absorption and dissolution; these nanocrystals do not need a carrier; only stabilizers such as polymeric steric stabilizers or surfactants are added. Moreover, in addition to oral administration, nanocrystal drug formulations can be administered through the parenteral, ocular, pulmonary and dermal routes [8].

An estimated $40 \%$ of new, approved medications and $90 \%$ of drugs in the research or discovery phase are poorly soluble in water [9]. Drug nanocrystals, nanosuspensions, and combined simultaneous transport of hydrophilic and hydrophobic drug molecules can overcome the problem of solubility - nanosization increases drug dissolution and bioavailabilty; a smaller dose of the drug is required, which reduces toxicity as well [10].

NPs have significantly greater bioavailability because of 
their nano size - NPs that measure between $10 \mathrm{~nm}$ and 200 $\mathrm{nm}$ persist for much longer in the bloodstream, largely avoiding filtration by the kidneys, liver and spleen [11]. For example, a nanosuspension of the antibiotic tinidazole showed higher dissolution and oral bioavailability; it was also more palatable to the patient [12].

Apart from size, the shape and texture of NPs can also be used in immune modulation. Spherical NPs, for instance, have greater macrophage uptake [13] and, when injected, textured polymeric NPs recruit neutrophils faster and enable easier phagocytosis at the injection site [14]. Lipid nanoparticles (LNPs) can mimic natural membranes. In one study, segments of CD47, a peptide recognized by the immune system as "self", was synthetically designed and attached to LNPs, resulting in reduced clearance by macrophages and a much longer circulation time[15].

In the treatment of highly lethal brain tumors like glioblastomas, the blood-brain barrier (BBB) presents a considerable challenge to orally administered chemotherapeutic agents. Alternative delivery routes like intranasal routes bypass the $\mathrm{BBB}$ and also reduce systemic toxicity [16]. However, drugs can be degraded by enzymes or eliminated by mucociliary action, and there may be difficulty in penetrating the nasal mucosa. Nanocarriers can solve these problems. Drugs trapped and encapsulated in nanoemulsions, polymeric NPs, and LNPs show great promise in enhancing intranasal delivery [17]. An additional advantage is that NPs with modified surfaces can target receptors and increase the specificity of drug delivery to glioblastomas [18].

Amphiphilic macromolecules have a hydrophobic and a hydrophilic component. A critical packing factor parameter (Cpp), enables these molecules to be developed into various nano architectures. At lower Cpp values, they form spherical and cylindrical micelles, vesicles, and lamellar structures. Micelles and vesicles (liposomes) offer the possibility of a variety of functions and combination therapies [19]. Nanoliposomal carriers can cross the BBB and have the potential for intracellular delivery. Their special structure enables them to deliver both hydrophilic and hydrophobic drugs.

Nanoliposomal carriers are effective and efficient drug carriers that have a low toxicity. They are nonimmunogenic, biocompatible, and biodegradable. Their other valuable attributes include the ability to stabilize the drug by encapsulating it, augmenting the drug's efficacy, and improving its therapeutic index [20] [21]. Doxorubicin, vincristine, cisplatin, and daunorubicin are among the anticancer drugs that have been used to investigate liposomes. Some of these have been used clinically in nanoliposome formulations [22].

Biodegradable polymers are potentially important nanocarriers that can facilitate targeted drug delivery [23]. These polymers can be designed as nanocarriers that release a drug to a targeted site and subsequently degrade into nontoxic products. They can also be engineered to selectively release their payload to specific sites only when triggered by external inducers such as light, magnetic fields, and ultrasound [24].

Dendrimers are smart nanocarriers that can deliver drugs selectively and safely to tumor cells. It is possible to control and shape the architecture of dendrimers to create optimal, spherical carriers for targeted drug delivery [25].

Photothermal therapy (PTT) and photodynamic therapy (PDT) are light-based therapies that more selectively target malignant tissues and therefore have fewer side effects than radiotherapy and chemotherapy. PTT shows the most promise in the treatment of tumors [26]. To kill every malignant cell, it needs to reach temperatures of 50 degrees $\mathrm{C}$ or more at the center of the tumo [27].

Many nanomaterials absorb light in the near-infrared (NIR) range - the same spectrum used in PTT. They can act as photothermal absorbers, which means less light is needed to achieve optimal temperatures in the tumor, and less heat leaks out. This increases the photothermal effect where it is needed while reducing or eliminating any damage to normal cells. Colloidal gold nanoparticles (GNPs), in the form of nanorods, nanocages, or nanoshells, can be modified, refined, and used with PTT to absorb specific wavelengths of light, giving GNPs exceptional specificity [26].

Unfortunately, heat can leak outwards and damage healthy surrounding tissue as well. Gold NPs (AuNPs), in conjunction with PTT, offers heating in a localized, targeted, tumor-specific manner, thereby safeguarding healthy tissues around the tumor. AuNPs can form conjugates with molecules like siRNAs (small interfering RNA), delivering the nucleic acid into the cell, where it remains inert until triggered by PTT. Once activated, the nucleic acid acts on the tumor cell's messenger RNA to degrade it and halt the production of tumor-associated proteins [28]. Graphene oxide (GO) nanosheets [29] and single-walled carbon nanotubes (SWCNT) [30] have also been utilized in combination with PTT for cancer treatment.

Tumor biomarkers are used to diagnose cancer, monitor the efficacy of therapeutic interventions, and assess the risk of developing certain cancers. They are of value in not only diagnosing cancers but also monitoring the response to treatment. For instance, AFP and hormones like beta human chorionic gonadotropin (bHCG) are elevated in patients with germ cell testicular tumors (GCTs) but decrease in response to treatment [31].

Nanomaterials have the ability to bind with biomarkers to diagnose cancer with a higher sensitivity and specificity. Quantum dots and gold nanoparticles are NPs that look promising in this area [32]. Polymer dots are also being investigated. The relatively large surface of an NP can be covered with antibodies, aptamers, peptides, and the like, to more easily recognize tumor-specific molecules [33].

Nanomaterials can detect tumor biomarkers like proteins, circulating tumor DNA, microRNA, and extracellular vesicles. They can detect tumor cells based on cell surface proteins, mRNA, and circulating tumor cells (CTCs). They can measure many targets simultaneously and can also be used for imaging in vivo [33].

\section{THERANOSTICS IN CANCER THERAPY}

Theranostics is a new branch of medicine based on nanoscience, a combination of therapeutics and diagnostics. A single agent is used to diagnose and treat diseases like cancers and also monitor the response to treatment. Theranostics uses nanotechnology for both imaging and 
drug delivery, assembling molecular platforms to diagnose and treat cancers - a therapeutic strategy known as nanotheranostics [34].

Some nanomedicine-based therapeutic agents can be combined with traditional chemotherapy to enhance efficacy. They can also be combined into a single formulation for superior treatment outcomes. Examples are enzymes, catalysts, genes, hypoxia-activated molecules, inorganic nanoparticles, and photothermal and photosensitization therapies [35].

NPs based on hyaluronic acid (HA-NPs) are advanced nanoplatforms for imaging and cancer therapy. They can be modified to suit various theranostic strategies and amended into different nanoformulations. Chitosan-based nanoparticles also show considerable promise as theranostic agents in both imaging and therapy for cancers [36]. Chitosan functionalized magnetic graphene NPs are another promising theranostic platform.

Known as CMG NPs, they allow the delivery of superparamagnetic iron oxide, genes and drugs at the same time, enabling both imaging and cancer therapy [37]. Iron oxide nanoparticle-modified graphene oxide nanoparticles (GO-IONP) have been investigated in conjunction with PTT to determine possible applications in cancer theranostics [38]. In the tumor tissue, GO sheets serve as photothermal absorbers that refine and enhance PTT, while iron oxide crystals serve as imaging contrast for MRIs.

Photoacoustic imaging [27], positron emission computed tomography [39], and fluorescence imaging [40] are other possible theranostic applications that use nanomaterials in combination with PTT.

Theranostics is an important step in the quest to make cancer nanomedicine more personalized and precise, in contrast to traditional avenues that take more of a trial and error, one-size-fits-all approach.

\section{TUMOR HETEROGENEITY AND CRISPR/CAS}

Tumors exhibit different attributes at the cellular and even molecular level. Diversity and mutations in tumor cell lines can be seen within a single tumor; tumors of the same broad type also change their cellular characteristics from patient to patient. This tumor heterogeneity is an important determinant of treatment failures in cancer therapy [41]. It is common in all varieties of cancer, from the level of the tumor cell population to the position of subpopulations in regions and tissues, to the epigenetic and genetic level [42]. Current research suggests that the renewal of stem cells [43] as well as genetic instability [44] cause tumor heterogeneity. Cell to cell interactions (tumor to host and tumor to tumor), cell to matrix interactions, and even interactions between cells and therapeutic agents can have an impact on tumor heterogeneity. For example, chemotherapy itself can sometimes contribute to heterogeneity [45].

Cancer is complex and dynamic because of tumor heterogeneity, which not only ensures the development and progression of neoplasms, but also enables immune escape and resistance to treatment. It is the main reason why cancers require multiple modalities of treatment; it limits or lowers the efficacy of targeted therapies and compromises therapeutic outcomes [46]. Therefore, treatment often fails, especially when administered in the later stages of the disease. For instance, single-agent targeted therapies are unlikely to have the intended lethal effect on tumors.

Molecular aberrations can occur in the hundreds or even thousands in a single tumor. CRISPR (clustered, regularly interspaced, short palindromic repeat) systems can detect the mutations present in a given cancer. The challenge is to reliably identify which of these mutations are driving the development and progression of the tumor. Individual patients may have mutations in unique combinations, which can have a significant impact on tumor growth patterns, metastasis, and sensitivity to treatment [47].

CRISPR is a faster and less expensive method that can also model multi-phase, complex mutations associated with aberrant cancer phenotypes [48]. CRISPR systems have, however, raised concerns about its use in vivo, including its efficiency and specificity, as well as safe and viable methods of delivery [49]. Thus far, viral vectors have been the main delivery mechanism for successful CRISPR editing; however, target-specific customization with these vectors is labor-intensive, and clinical challenges include immune reactions and the possibility of uncommon but dangerous integrations [50].

CRISPR, when used with CRISPR-associated proteins (Cas) in the CRISPR/Cas system, allows highly specific genome and epigenome editing. CRISPR/Cas has been widely adopted to explore the molecular basis of various types of cancer. It edits tumor-related oncogenes with accuracy, acts at the junction of genetic and biochemical interactions, provide insights into how tumor cells respond to drugs, and modify beneficial viruses and immune cells for use in cancer immunotherapy [51]. In addition, CRISPR/dCas9 (dead or deactivated Cas9) allows sitespecific epigenetic edits and functional modulation of genes. Both systems can down-regulate the expression of target genes, but CRISPR/dCAS9 is significantly more consistent and efficient [52].

If CRISPR/Cas systems can be delivered safely through nonviral means that allow large-scale production, it would greatly enhance the therapeutic utility of these systems. Polymer- and lipid-based nanocarriers hold great promise for the safe and efficient delivery of CRISPR/Cas [53]. A new class of zwitterionic amino lipids (ZALs), used as a ZAL nanoparticle (ZNP), enables permanent DNA editing in vivo and holds promise in enhancing the safety and increased clinical utility of gene editing [50].

Although the last few decades have seen the deployment of several nonviral vectors in gene therapy, few are used to deliver CRISPR/Cas9. This is, perhaps, because of the twin challenges of encapsulating the relatively large Cas9 protein and delivering CRISPR/Cas9 to the nucleus to commence the process of genome editing. Lipid-based cationic vectors are an effective method of delivery; however, this is usually restricted to local administration [53].

Multistage delivery nanoparticles (MDNP) could provide the answer to these delivery challenges. They have a coreshell design, where the shell is a polymer with responsive properties. Thus, MDNP can change their surface in response to the environment, enabling them to cross multiple physiological barriers to deliver the required payloads that target tumor cells [54]. 
Although biomaterials and genome editing are evolving rapidly, their great promise remains elusive in practical, clinical settings, for now. However, there has been significant progress in correcting pathologies in the genome sequence of human cells such as $\mathrm{T}$ cells, which could have therapeutic applications in cancer immunotherapy in future. Genome editing could potentially be completed and validated before transplanting cells back to the patient [55].

Extraordinary advances in delivery systems have also paved the way for new opportunities such as RNA-guided CRISPR/Cas delivery ex vivo and tissue-specific delivery in vivo [56] .

\section{TUMOR IMMUNOSUPPRESSION}

The immune system can identify, target, and eliminate malignant tumors, bacteria, viruses, antigens and foreign bodies. Malignant tumors can suppress immune surveillance through a variety of strategies. For example, they can avoid the expression of tumor-specific antigens on the cell surface, thus escaping immune recognition, and alter the tumor microenvironment (TME) to make it much more immune tolerant [57].

Malignant cells alter the TME by (i) secreting an array of molecules that suppress immunity, including prostaglandin E2, TGF- $\beta$, IL-10 and VEGF [58], (ii) expressing immune checkpoint inhibitors such as CTLA-4, PD-L1, and Vdomain immunoglobulin suppressor of $\mathrm{T}$-cell activation (VISTA) [59], and (iii) using tumor-derived chemokines to recruit immunosuppressive cells like tumor-associated macrophages (TAMs), myeloid-derived suppressor cells (MDSC), and T regulatory cells (Tregs) [60] [61]. Besides, tumor cells can defend themselves by inhibiting phagocytosis through an over-expression of CD47, the transmembrane ligand for Signal Regulatory Protein $\alpha$ $(\mathrm{SIRP} \alpha)$ in macrophages and dendritic cells [62] .

\section{IMMUNOTHERAPY}

\section{A. Adoptive T-Cell Therapy}

Nanotechnology is currently used to enhance the function, specificity, and localization of the body's inherent $\mathrm{T}$ cells as well as transferred T cells [63]. Genetically modified T cells are a new and promising class of cancer therapies. Immune cells, usually $\mathrm{T}$ cells, are modified to express CARs (Chimeric Antigen Receptors) that target a specific antigen. $\mathrm{T}$ cells engineered in this way are known as CAR $\mathrm{T}$ cells [64].

The patient's $\mathrm{T}$ cells are harvested, engineered in a laboratory, and infused back into the patient, where they proliferate and directly kill many malignant cells. This is a "living therapy" because CAR T cells have to only be infused once; from that point, they continue to proliferate and may even become more efficacious over time. They may play a role in augmenting immune surveillance and preventing tumor recurrence by releasing antigens and assisting lymphocytes.

However, this remarkable new therapy has serious side effects that are potentially lethal. Over-stimulation of the immune system can cause Cytokine Release Syndrome
(CRS), which occurred in $57 \%$ to $97 \%$ of patients in clinical trials [65]. Multiple systems are involved; patients can have fulminant hemophagocytic lymphohistiocytosis (HLH), ARDS (acute respiratory distress syndrome), cardiomyopathy, arrythmias, AKI (acute kidney injury), and DIC (disseminated intravascular coagulation) [66].

CAR-T cell-related encephalopathy syndrome (CRES) is not uncommon. Symptoms and signs range from confusion, disorientation and dysphasia, to seizures and fatal cerebral edema [67]. CAR T cell therapy is already in use to treat leukemias and lymphomas [68]. There is evidence of its efficacy in even treating some of the most challenging solid tumors - malignant tumors of the brain, such as glioblastoma [69].

As this therapeutic pathway becomes more established, multi-disciplinary teams of specialists may be needed, to manage the potentially lethal side effects and multi-organ syndromes [65].

\section{B. Immune Checkpoint Inhibitors}

$\mathrm{T}$ cells are prominent members of the immune system's army of defenses against cancer [70]. Receptors on their cell surfaces, called immune checkpoints, control the immune response. They enable the immune system to differentiate between normal and 'foreign' cells, ensuring that $\mathrm{T}$ cells do not attack normal cells. Receptors such as the programmed cell death receptor 1 (PD-1) and cytotoxic T-lymphocyteassociated protein 4 (CTLA-4) act as "off switches" inhibitory immune signals that inactivate $\mathrm{T}$ cells until required [71].

On the other hand, the "on switch" or costimulatory pathway usually consists of two sequential activation signals. The first signal occurs when antigen-presenting cells (APCs) or major histocompatibility complex (MHC) molecules on tumor cells present antigens to $\mathrm{T}$ cell receptors [72]. The second signal occurs when the B7 family of signaling molecules (ligands) on dendritic cells interact with the CD28 family of receptors on T cells [73].

Tumor cells can hijack these checkpoints to avoid immune surveillance or effect immune escape. For example, some tumors turn down the $\mathrm{T}$ cell response by producing quantities of a protein known as Programmed Cell Death Ligand 1 (P D-Li) that acts as a co-inhibitory factor along with PD-1 [74].

Immune checkpoint inhibitors (ICIs) are drugs that target inhibitory receptors on the surface of $\mathrm{T}$ cells to reactivate the immune system's anti-tumor response [75] [76]. ICIs prevent immune checkpoints from triggering the "off" switch, allowing $\mathrm{T}$ cells to remain active and kill tumor cells. Therefore, ICIs augment the immune system's natural response to cancers, enabling it to develop a more robust immune memory, preventing recurrences, and aiding the patient's long-term survival [77]. ICIs like ipilimumab act as anti-CTLA-4 antibodies, whereas other ICIs such as pembrolizumab and nivolumab act as anti-PD-1 antibodies.

ICIs were initially approved for the treatment of melanoma, but these remarkable drugs are now approved for the treatment of many other cancers, including cervical, liver, bladder, and colon cancers, as well as Hodgkin's lymphoma. ICIs that act on PD-1, PD-L1, and CTLA-4 have been approved for use in the US. They prevent immune 
evasion and immune escape by tumor cells [78].

At least $20 \%$ of patients with advanced melanoma who received ipilimumab survived long-term [79]. 15\% of those who received pembrolizumab had a complete and durable immune response even after the drug was discontinued [80]. Pretreated patients who received nivolumab achieved enhanced long-term survival in renal cell carcinoma, advanced melanoma, and non-small cell lung cancer [81].

Nanoparticles (NPS) can improve the outcome of combination therapies. NPs enhance the co-delivery of adjuvants and antigens, thereby augmenting the anti-tumor immune response [82]. Antigen-capturing nanoparticles (AC-NPs) may augment RT-ICI combination therapy for metastatic cancers [83]. Novel combinations of ICIs with other immunotherapies, targeted therapy, and/or radiation therapy (RT) are being investigated [84]. Where immunotherapy has been limited by tumor heterogeneity, nanotechnologies like AC-NPs may be a valuable addition to novel therapeutic combinations. For example, they can expose the immune system to a far greater range of antigens, increasing the abscopal effect of the RT-ICI combination [85]. ICIs used in combination with RT, cancer treatment vaccines, and nanomaterials may well pave the way to far better outcomes in cancer therapy.

\section{Monoclonal Antibodies}

Monoclonal antibodies (mAbs) have been investigated and developed for cancer therapy from the 1970s [86]. They are now considered important therapeutic agents, alongside chemotherapy, radiotherapy, and surgery. Whereas early efforts focused on tumor-associated antigens (TAAs), therapeutic strategies based on monoclonal antibodies have moved towards targeting immune cells in the TME to augment the immune response against tumor cells [87].

mAbs have wide ranging, clinically relevant mechanisms of action. They can kill target cells by complementdependent cytotoxicity, antibody-dependent cell cytotoxicity (ADCC), assisting macrophages antibody-dependent cellular phagocytosis (ADCP), and interfering with the signaling pathways of tumor cells [88]. These antibodies target tumor cells directly and also achieve long-term immune responses against tumors. For example, antibody therapy that targeted the $\mathrm{T}$ cell receptors CTLA-4, PD-1, and PD-L1, increased long-term survival in a significant percentage of patients [89].

Monoclonal antibodies are now used clinically to target tumors and target the TME directly. They are also used in antibody-drug conjugates (ADCs) and ICI therapies [90]. mAbs have a low level of toxicity and highly specific immunomodulatory actions. These desirable properties have forged the path to other efforts in nanomedicine [91].

\section{Cancer Treatment Vaccines}

Tumor cells have molecules on their cell surface, called cancer-specific antigens, which are not found in normal cells [92]. Cancer treatment vaccines may contain fragments, antigens, or whole tumor cells, which enhance the immune system's ability to recognize these antigens and destroy malignant cells [93]. Injected intradermally, these vaccines contain adjuvants that serve to activate dendritic cells (DCs); this is followed by antigen uptake by DCs, which present the antigen to CD4 and CD8 T cells to trigger them to act. CD8 cells then recognize the antigen on the surface of malignant cells and eliminate them [94].

Clinical trials with these vaccines are underway for the treatment of brain tumors, and bladder, breast, and cervical cancers [87]. However, cancer treatment vaccines suffer from the disadvantages of inefficient antigen presentation and DC uptake, and the rapid degradation and elimination of antigens. Nanomaterials enhance the efficacy of these vaccines by co-encapsulating and co-delivering both adjuvants and antigens, thus protecting against degradation and enhancing DC uptake [95].

\section{CONCLUSION}

Cancer nanomedicine has the potential and power to provide immune stimulation and targeted immune activation. The size, shape, and texture of nanomaterials enable unprecedented immune modulation that raises the hope of better outcomes even in challenging malignancies like advanced melanoma. The innovative therapeutic strategies of nanomedicine hold the promise of augmenting the patient's own immunity, fostering a greater immune memory to reduce recurrence and improve long-term survival.

There is, now, tremendous promise in nanoscale drug delivery systems, which can simultaneously carry drugs with disparate attributes across physiological barriers. Drug therapies need not be restricted by the food effect and absorption problems associated with oral administration. Nanocarriers herald an era where alternatives like intranasal administration may find their rightful place in the safe, efficacious delivery of anti-cancer drugs. Combination therapies based on immunotherapy complement conventional treatments like chemotherapy, radiotherapy and surgery, and may hold the key to more efficacious treatment protocols. Although there is some way to go on the long and winding road, there is no doubt that cancer nanomedicine is already delivering on its potential to unlock a brave new world.

\section{REFERENCES}

[1] S. Tinkle, S.E. McNeil, S. Mühlebach, R. Bawa, G. Borchard, Y Barenholz, L. Tamarkin and N. Desai, "Nanomedicines: addressing the scientific and regulatory gap," Annals of the New York Academy of Sciences, vol. 1313, pp. 35-56, April 2014, https://doi.org/10.1111/nyas.12403.

[2] D. Gao, X. Guo, X. Zhang, S. Chen, Y. Wang, T. Chen, G. Huang, Y. Gao, Z. Tian and Z. Yang, "Multifunctional phototheranostic nanomedicine for cancer imaging and treatment," Materials Today Bio, vol. 5, no. 100035, pp. 1-33, January 2020, https://doi.org/10.1016/j.mtbio.2019.100035.

[3] Jeevanandam, J, Barhoum, A, Chan, YS, Dufresne, A, and Danquah, MK 2018, "Review on nanoparticles and nanostructured materials: history, sources, toxicity and regulations," Beilstein Journal of Nanotechnology, vol. 9, pp. 1050-1074, April 2018 , https://doi.org/10.3762/bjnano.9.98.

[4] M. Rahm, R. Hoffmann and N.W. Ashcroft, "Atomic and ionic radii of elements 1-96," Chemistry: A European Journal, vol. 22, no. 41, pp. 14625-14632, August 2016.

[5] J. Szebeni and Y.C. Barenholz, "Complement activation, immunogenicity, and immune suppression as potential side effects of liposomes," in Handbook of harnessing biomaterials in nanomedicine - preparation, toxicity and applications, D. Peer, Ed. Singapore: Pan Stanford, 2012, ch. 11, pp. 309-335.

[6] D. Peer, J. M. Karp, S. Hong, O. C. Farokhzad, R. Margalit and R. Langer, "Nanocarriers as an emerging platform for cancer therapy," Nature Nanotechnology, vol. 2, no. 12, pp. 751-760, December 2007. 
[7] R.D. Toothaker and P.G. Welling, "The Effect of Food on Drug Bioavailability," Annual Review of Pharmacology and Toxicology, vol. 20, no. 9, pp. 173-199, February 1980, https://doi.org/10.1146/annurev.pa.20.040180.001133.

[8] V.B. Junyaprasert, and B. Morakul, "Nanocrystals for enhancement of oral bioavailability of poorly water-soluble drugs," Asian Journal of Pharmaceutical Sciences, vol. 10, no. 1, pp. 13-23, August 2014 https://doi.org/10.1016/j.ajps.2014.08.005.

[9] S. Kalepu and V. Nekkanti, "Insoluble drug delivery strategies: review of recent advances and business prospects," Acta Pharmaceutica Sinica B, vol. 5, no. 5, pp. 442-453, September 2015 , https://doi.org/10.1016/j.apsb.2015.07.003.

[10] S. Dizaj, Z. Vazifehasl, S. Salatin, K. Adibkia, and Y. Javadzadeh, "Nanosizing of drugs: effect on dissolution rate," Research in Pharmaceutical Sciences, vol. 10, pp. 95-108, Apr 2015.

[11] N. Hoshyar, S. Gray, H. Han, and G. Bao, "The effect of nanoparticle size on in vivo pharmacokinetics and cellular interaction," Nanomedicine (London), vol. 6, pp. 673-692, March 2016, https://doi.org/10.2217/nnm.16.5.

[12] R.N. Pawar S.N. Chavan, and M.D. Menon, "Development, characterization, and evaluation of tinidazole nanosuspension for treatment of amoebiasis," Journal of Nanomedicine and Nanotechnology, vol. 7, no. 6, pp. 1-4, January 2016 , https://doi.org/10.4172/2157-7439.1000413.

[13] R. Toy, P.M. Peiris, K.B. Ghaghada, and E. Karathanasis, "Shaping cancer nanomedicine: the effect of particle shape on the in vivo journey of nanoparticles," Nanomedicine (London, England), vol. 9 , no. 1, pp. 121-134, January 2014, https://doi.org/10.2217/nnm.13.191

[14] C.A. Vaine, M.K. Patel, J. Zhu, E. Lee, R.W. Finberg, R.C. Hayward, and E.A. Kurt-Jones, "Tuning innate immune activation by surface texturing of polymer microparticles: the role of shape in inflammasome activation," The Journal of Immunology, vol. 190, no. 7, pp. 3525-3532, $\quad$ February 2013 https://doi.org/10.4049/jimmunol.1200492.

[15] P.L. Rodriguez, T. Harada, D. Christian, D.A. Pantano, R.K. Tsai, and D.E. Discher, "Minimal "self" peptides that inhibit phagocytic clearance and enhance delivery of nanoparticles," Science, vol. 339, no. 6122, pp. 971-975, February 2013 https://doi.org/10.1126/science.1229568.

[16] F.A. Bruinsmann, G.R. Vaz, A. de C.S. Alves, T. Aguirre, A.R. Pohlmann, S.S. Guterres, and F. Sonvico, "Nasal drug delivery of anticancer drugs for the treatment of glioblastoma: preclinical and clinical trials," Molecules, vol. 24, no. 23, pp. 1-32, November 2019 , https://doi.org/10.3390/molecules24234312.

[17] C. Comfort, G. Garrastazu, M. Pozzoli, and F. Sonvico, "Opportunities and challenges for the nasal administration of nanoemulsions," Current Topics in Medicinal Chemistry, vol. 15, no. 4, pp. 356-368, January 2015 https://doi.org/10.2174/1568026615666150108144655.

[18] L. Chu, A. Wang, L. Ni, X. Yan, Y. Song, M. Zhao, K. Sun, H. Mu, S. Liu, Z. Wu, and C. Zhang, "Nose-to-brain delivery of temozolomide-loaded PLGA nanoparticles functionalized with antiEPHA3 for glioblastoma targeting," Drug Delivery, vol. 25, no. 1, pp 1634-1641, September https://doi.org/10.1080/10717544.2018.1494226.

[19] D. Lombardo, M.A. Kiselev, and M.T. Caccamo, "Smart nanoparticles for drug delivery application: development of versatile nanocarrier platforms in biotechnology and nanomedicine," Journal of Nanomaterials, vol. 2019, no. 3702518, pp. 1-26, February 2019 , https://doi.org/10.1155/2019/3702518.

[20] A. Akbarzadeh, R. Rezaei-Sadabady, S. Davaran, SW. Joo, N Zarghami, Y. Hanifehpour, M. Samiei, M. Kouhi, and K. NejatiKoshki, "Liposome: classification, preparation, and applications," Nanoscale Research Letters, vol. 8, no. 1, p. 102, February 2013 , https://doi.org/10.1186/1556-276X-8-102.

[21] L. Dutta, B. Mukherjee, T. Chakraborty, M.K. Das, L. Mondal, S. Bhattacharya, R.H. Gaonkar, and M.C. Debnath, "Lipid-based nanocarrier efficiently delivers highly water soluble drug across the blood-brain barrier into brain," Drug Delivery, vol. 25, no. 1, pp. 504 516 , February https://doi.org/10.1080/10717544.2018.1435749.

[22] G. Bozzuto, and A. Molinari, "Liposomes as nanomedical devices," International Journal of Nanomedicine, vol. 10, pp. 975-999, February 2015, https://doi.org/10.2147/IJN.S68861.

[23] P.J.J. Francis, K.J. Arun, A.A. Navas, and I. Joseph, "Biomedical applications of polymers - an overview," Current Trends in Engineering and Biosciences, vol. 15, no. 2, pp. 44-45, June 2018, https://doi.org/10.19080/ctbeb.2018.15.555909.

[24] J. Karlsson, H.J. Vaughan, and J.J. Green, "Biodegradable polymeric nanoparticles for therapeutic cancer treatments," Annual Review of
Chemical and Biological Engineering, vol. 7, no. 9, pp. 105-127, June 2018, https://doi.org/10.1146/annurev-chembioeng-060817-084055.

[25] P. Anitha, J. Bhargavi, G. Sravani, B. Aruna, and S. Ramkanth, "Recent progress of dendrimers in drug delivery for cancer therapy," International Journal of Applied Pharmaceutics, vol. 10, pp. 34-42, September 2018, https://doi.org/10.22159/IJAP.2018V10I5.27075.

[26] A.C.V. Doughty, A.R. Hoover, E. Layton, C.K. Murray, E.W Howard, and W.R. Chen, "Nanomaterial applications in photothermal therapy for cancer," Materials (Basel), vol. 12, no. 1, p. 779, March 2019, https://doi.org/10.3390/ma12050779.

[27] C. Zhang, W. Bu, D. Ni, C. Zuo, C. Cheng, Q. Li, L. Zhang, Z. Wang, and J. Shi, "A polyoxometalate cluster paradigm with self-adaptive electronic structure for acidity/reducibility-specific photothermal conversion," Journal of the American Chemical Society, vol. 138, no. 26, pp. 8156-8164, June 2016, https://doi.org/10.1021/jacs.6b03375.

[28] F.M. Kouri, L.A. Hurley, W.L. Daniel, E.S. Day, Y. Hua, L. Hao, C.Y. Peng, T.J. Merkel, M.A. Queisser, C. Ritner, H. Zhang, C.D James, J.I. Sznajder, L.Chin, D.A. Giljohann, J.A. Kessler, M.E Peter, C.A. Mirkin, and A.H. Stegh, "miR-182 integrates apoptosis, growth, and differentiation programs in glioblastoma," Genes and Development, vol. 29, no. 7, pp. 732-45, April 2015, https://doi.org/10.1101/gad.257394.

[29] K. Yang, J. Wan, S. Zhang. B, Tian. Y. Zhang, and Z. Liu, "The influence of surface chemistry and size of nanoscale graphene oxide on photothermal therapy of cancer using ultra-low laser power,' Biomaterials, vol. 33, no. 7, pp. 2206-2214, December 2011, https://doi.org/10.1016/j.biomaterials.2011.11.064.

[30] X. Ma, H. Tao, K. Yang, L. Feng, L. Cheng, X. Shi, Y. Li, L. Guo, and Z. Liu, "A functionalized graphene oxide-iron oxide nanocomposite for magnetically targeted drug delivery, photothermal therapy, and magnetic resonance imaging," Nano Research, vol. 5, pp. 199-212, March 2012, https://doi.org/10.1007/s12274-012-0200-

[31] K-P. Dieckmann, H. Simonsen-Richter, M. Kulejewski, P. Anheuser, H. Zecha, H. Isbarn, and U. Pichlmeier, "Serum tumour markers in testicular germ cell tumours: frequencies of elevated levels and extents of marker elevation are significantly associated with clinical parameters and with response to treatment," BioMed Research International, vol. 2019, no. 5030349, pp. 1-22, May 2019 , https://doi.org/10.1155/2019/5030349.

[32] Y-E. Choi, J-W. Kwak, and J.W. Park, "Nanotechnology for Early Cancer Detection," Sensors, vol. 10, pp. 428-455, January 2010 , https://doi.org/10.3390/s100100428.

[33] Y. Zhang, M. Li, X. Gao, Y. Chen, and T. Liu, "Nanotechnology in cancer diagnosis: progress, challenges and opportunities," Journal of Hematology and Oncology, vol. 12, no. 137, pp. 1-13, December 2019, https://doi.org/10.1186/s13045-019-0833-3.

[34] K.J. Arun, A.A. Navas and P.J.J. Francis, "Novel trends in the management of Alzheimer's disease by using nano based materials," Madridge Journal of Nanotechnology and Nanoscience, vol. 3, no. 1 , pp. 96-97, April 2018, https://doi.org/10.18689/mjnn-1000118.

[35] S. Yu, Z. Chen, X. Zeng, X. Chen and Z. Gu, "Advances in nanomedicine for cancer starvation therapy," Theranostics, vol. 9, no. 26, pp. 8026-8047, October 2019, https://doi.org/10.7150/thno.38261.

[36] M. Swierczewska, H.S. Han, K. Kim, J.H. Park, and S. Lee, "Polysaccharide-based nanoparticles for theranostic nanomedicine," Advanced Drug Delivery Reviews, vol. 99, part A, pp. 70-84, April 2016, https://doi.org/10.1016/j.addr.2015.11.015.

[37] C. Wang, S. Ravi, U.S. Garapati, M. Das, M. Howell, J. Mallela, S Alwarappan, S.S. Mohapatra, and S. Mohapatra, "Multifunctional chitosan magnetic-graphene (CMG) nanoparticles: a theranostic platform for tumor-targeted co-delivery of drugs, genes and MRI contrast agents," Journal of Materials Chemistry B, vol. 1, pp. 43964405, June 2013, https://doi.org/10.1039/C3TB20452A

[38] S. Wang, Q. Zhang, X.F. Luo, J. Li, H. He, F. Yang, Y. Di, C. Jin, X.G. Jiang, S. Shen, and de L. Fu, "Magnetic graphene-based nanotheranostic agent for dual-modality mapping guided photothermal therapy in regional lymph nodal metastasis of pancreatic cancer," Biomaterials, vol. 35, no. 35, pp. 9473-83, August 2014, https://doi.org/10.1016/j.biomaterials.2014.07.064.

[39] L. Cui, C. Xiong. M, Zhou. S, Shi. D.S. Chow, and C. Li, "Integrin avß3-targeted $\mathrm{CuS}$ nanoparticles for PET/CT imaging and photothermal ablation therapy," Bioconjugate Chemistry, vol, 29, no, 12, pp. 4062-4071, November 2018 , https://doi.org/10.1021/acs.bioconjchem.8b00690.

[40] X. Zhen, J. Zhang, J. Huang, C. Xie, Q. Miao, and K. Pu, "Macrotheranostic probe with disease-activated near-infrared fluorescence, photoacoustic, and photothermal signals for imagingguided therapy," Angewandte Chemie Internal Edition English, no. 
57, no. 26, pp. 7804-7808, June 2018 https://doi.org/10.1002/anie.201803321.

[41] S.R. y Cajal, M. Sesé, C. Capdevila, T. Aasen, De L. Mattos-Arruda, S.J. Diaz-Cano, J. Hernández-Losa, and Castellví. J. "Clinical implications of intratumor heterogeneity: challenges and opportunities," Journal of Molecular Medicine, vol. 98, no. 2, pp. 161-177, February 2020, https://doi.org/10.1007/s00109-020-018742.

[42] D.R. Welch, "Tumor heterogeneity - a 'contemporary concept' founded on historical insights and predictions," Cancer Research, vol 76, no. 1, pp. 4-6, January 2016, https://doi.org/10.1158/00085472.CAN-15-3024.

[43] A. Skibinski and C. Kuperwasser, "The origin of breast tumor heterogeneity," Oncogene, vol. 34, no. 5, pp. 5309-5316, October 2015, https://doi.org/10.1038/onc.2014.475

[44] S. Huang, "Genetic and non-genetic instability in tumor progression: link between the fitness landscape and the epigenetic landscape of cancer cells," Cancer Metastasis Review, vol. 32, no. 3-4, pp. 423448, December 2013, https://doi.org/10.1007/s10555-013-9435-7.

[45] T.J. McMillan and I.R. Hart, "Can cancer chemotherapy enhance the malignant behaviour of tumours?," Cancer and Metastasis Reviews, vol.6, no. 4, pp. 503-520, February 1987, https://doi.org/10.1007/BF00047465.

[46] F. Janku, "Tumor heterogeneity in the clinic: is it a real problem?", Therapeutic Advances in Medical Oncology, vol. 6, no. 2, pp. 43-51, March 2014, https://doi.org/10.1177/1758834013517414.

[47] R.D. Chow and S. Chen, "Cancer CRISPR screens in vivo," Trends Cancer, vol. 4, no. 5, pp. 349-358, May 2018 , https://doi.org/10.1016/j.trecan.2018.03.002.

[48] A. Guernet and L. Grumolato, "CRISPR/Cas9 editing of the genome for cancer modeling," Methods, vol. 121-122, pp. 130-137, May 2017, https://doi.org/10.1016/j.ymeth.2017.03.007.

[49] C. Moses, B. Garcia-Bloj, A.R. Harvey, and P. Blancafort, "Hallmarks of cancer: The CRISPR generation," European Journal of Cancer, vol. 93, pp. 10-18, April 2018, https://doi.org/10.1016/j.ejca.2018.01.002.

[50] J.B. Miller, S. Zhang, P. Kos, H. Xiong, K. Zhou, S.S. Perelman, H. Zhu, and D.J. Siegwart, "Nonviral CRISPR/Cas gene editing in vitro and in vivo enabled by synthetic nanoparticle co-delivery of Cas9 mRNA and sgRNA," Angewandte Chemie International Edition, vol. 56, pp. 1059-1063, December 2016, https://doi.org/10.1002/anie.201610209.

[51] B. Liu, A. Saber, and H.J. Haisma, "CRISPR/Cas9: a powerful tool for identification of new targets for cancer treatment," Drug Discovery Today, vol. 24, no. 4, pp. 955-970, April 2019, https://doi.org/10.1016/j.drudis.2019.02.011.

[52] X. Dai, X. Chen, Q. Fang, J. Li, and Z. Bai, "Inducible CRISPR genome-editing tool: classifications and future trends," Critical Reviews in Biotechnology, vol. 38, no. 4, pp. 573-586, June 2018, https://doi.org/10.1080/07388551.2017.1378999.

[53] L. Li, S. Hu, and X. Chen, "Nonviral delivery systems for CRISPR/Cas9-based genome editing: Challenges and opportunities," Biomaterials, vol. 171, pp. 207-218, July 2018 https://doi.org/10.1016/j.biomaterials.2018.04.031.

[54] Q. Liu, K. Zhao, C. Wang, Z. Zhang, C. Zheng, Y. Zhao, Y. Zheng, C. Liu, Y. An, L. Shi, C. Kang, and Y. Liu, "Multistage delivery nanoparticle facilitates efficient CRISPR/dCas9 activation and tumor growth suppression in vivo," Advanced Science, vol. 6, no. 1, pp. 112, October 2018, https://doi.org/10.1002/advs.201801423.

[55] K. Schumann, S. Lin, E. Boyer, D.R. Simeonov, M. Subramaniam, R.E. Gate, G.E. Haliburton, C.J. Ye, J.A. Bluestone, J.A. Doudna, and A. Marson, "Generation of knock-in primary human T cells using Cas9 ribonucleoproteins," Proceedings of the National Academy of Sciences of the United States of America, vol. 112, no. 33, pp. 1043710442, July 2015, https://doi.org/10.1073/pnas.1512503112.

[56] M.L. Maeder, and C.A. Gersbach, "Genome-editing Technologies for Gene and Cell Therapy," Molecular Therapy, vol. 24, no. 3, pp. 430446, January 2016, https://doi.org/10.1038/mt.2016.10.

[57] H. Gonzalez, C. Hagerling, and Z. Werb, "Roles of the immune system in cancer: from tumor initiation to metastatic progression,' Genes and Development, vol. 32, no. 19-20, pp. 1267-1284, October 2018, https://doi.org/10.1101/gad.314617.118.

[58] B. Burkholder, R-Y. Huang, R. Burgess, S. Luo, V.S. Jones, W. Zhang, Z-Q. Lv, C-Y. Gao, B-L. Wang, Y-M. Zhang, and R-P. Huang, "Tumor-induced perturbations of cytokines and immune cell networks," Biochimica et Biophysica Acta (BBA) - Reviews on Cancer, vol. 1845, no.2, pp. 182-201, April 2014, https://doi.org/10.1016/j.bbcan.2014.01.004.

[59] M.A. ElTanbouly, W. Croteau, R.J. Noelle, and J.L. Lines, "VISTA: a novel immunotherapy target for normalizing innate and adaptive immunity," Seminars in Immunology, vol. 42, no. 101308, pp. 1-14, April 2019, https://doi.org/10.1016/j.smim.2019.101308.

[60] A. Mantovani, F. Marchesi, A. Malesci, L. Laghi, and P. Allavena, "Tumour-associated macrophages as treatment targets in oncology," Nature Reviews Clinical Oncology, vol. 1, no. 7, pp. 399-416, July 2017, https://doi.org/10.1038/nrclinonc.2016.217.

[61] A. Tanaka, and S. Sakaguchi, "Regulatory $T$ cells in cancer immunotherapy," Cell Research, vol. 27, no.1, pp. 109-118, January 2017, https://doi.org/10.1038/cr.2016.151.

[62] M.A. Smolle, and M. Pichler, "Inflammation, phagocytosis and cancer: another step in the CD47 act," Journal of Thoracic Disorders, vol. 9, no. 8, pp. 2279-2282, August 2017, https://doi.org/10.21037/jtd.2017.07.38.

[63] M.S. Goldberg, "Improving cancer immunotherapy through nanotechnology," Nature Reviews Cancer, vol. 19, pp. 587-602, October 2019, https://doi.org/10.1038/s41568-019-0186-9.

[64] M. Kalos, B.L. Levine, D.L, Porter, S. Katz, S.A. Grupp, A. Bagg, and C.H. June, "T Cells with chimeric antigen receptors have potent antitumor effects and can establish memory in patients with advanced leukemia," Science Translational Medicine, vol. 3, no. 95, pp. 95-73, August 2011, https://doi.org/10.1126/scitranslmed.3002842.

[65] C. Graham, R. Hewitson, A. Pagliuca, and B. Reuben "Cancer immunotherapy with CAR-T cells - behold the future," Clinical Medicine, vol. 18, no. 4, pp. 324-328, August 2018 , https://doi.org/10.7861/clinmedicine.18-4-324.

[66] S.S. Neelapu, S. Tummala, P. Kebriaei, W. Wierda, C. Gutierrez, F.L. Locke, K.V. Komanduri, Y. Lin, N. Jain, N. Daver, J. Westin, A.M Gulbis, M.E. Loghin, J.F. de Groot, S. Adkins, S.E. Davis, K Rezvani, P. Hwu. and E.J. Shpall, "Chimeric antigen receptor T-cell therapy - assessment and management of toxicities," Nature Reviews Clinical Oncology, vol, 15, no. 1, pp. 47-62, January 2018 , https://doi.org/10.1038/nrclinonc.2017.148.

[67] M.L. Davila, I. Riviere, X. Wang, S. Bartido, J. Park, K. Curran, S.S Chung, J. Stefanski, O. Borquez-Ojeda, M. Olszewska, J. Qu, T. Wasielewska, Q. He, M. Fink, H. Shinglot, M. Youssif, M. Satter, Y Wang, J. Hosey, H. Quintanilla, E. Halton, Y. Bernal, D.C Bouhassira, M.E. Arcila, M. Gonen, G.J. Roboz, P. Maslak, D. Douer, M.G. Frattini, S. Giralt, M. Sadelain, and R. Brentjens, "Efficacy and toxicity management of $19-28 \mathrm{z}$ CAR T cell therapy in B cell acute lymphoblastic leukemia," Science Translational Medicine, vol. 6, no 224, pp. 224-25, February 2014 https://doi.org/10.1126/scitranslmed.3008226.

[68] C.H. June and M. Sadelain, "Chimeric Antigen Receptor Therapy," The New England Journal of Medicine, vol. 379, pp. 64-73, July 2018, https://doi.org/10.1056/NEJMra1706169.

[69] D. Akhavan, D. Alizadeh, D. Wang, M.R. Weist, J. Shepphird, and C.E. Brown, "CAR T cells for brain tumors: Lessons learned and road ahead," Immunological Reviews, vol. 290, no. 1, pp. 60-84, July 2019 , https://doi.org/10.1111/imr.12773.

[70] S.Y. Gun, S.W.L. Lee, J.L. Sieow, and S.C. Wong, "Targeting immune cells for cancer therapy," Redox Biology, vol. 25, no. 101174, pp. 1-16, $\quad$ March 2019 , https://doi.org/10.1016/j.redox.2019.101174

[71] J. Seidel, A. Otsuka, and K. Kabashima, "Anti-PD-1 and anti-CTLA4 therapies in cancer: Mechanisms of action, efficacy, and limitations," Frontiers in Oncology, vol. 8, no. 86, pp. 1-14, March 2018, https://doi.org/10.3389/fonc.2018.00086.

[72] E. Hui, "Immune checkpoint inhibitors," Journal of Cell Biology, vol. 218, no. 3, pp. 740-741, February 2019 , https://doi.org/10.1083/jcb.201810035.

[73] A. Rotte, J.Y. Jin, and V. Lemaire, "Mechanistic overview of immune checkpoints to support the rational design of their combinations in cancer immunotherapy," Annals of Oncology, vol. 29, no. 1, pp. 7183, January 2018, https://doi.org/10.1093/annonc/mdx686.

[74] Y. Han, D. Liu, and L. Li, "PD-1/PD-L1 pathway: current researches in cancer," American Journal of Cancer Research, vol. 10, no. 3, pp. 727-742, March 2020

[75] J.J. Havel, D. Chowell, and T.A. Chan, "The evolving landscape of biomarkers for checkpoint inhibitor immunotherapy," Nature Reviews Cancer, vol. 19, no. 3, pp. 133-150, March 2019, https://doi.org/10.1038/s41568-019-0116-x.

[76] C. Robert, "A decade of immune-checkpoint inhibitors in cancer therapy," Nature Communications, vol. 11, no. 3801, pp. 1-3, July 2020, https://doi.org/rg/10.1038/s41467-020-17670-y.

[77] G.K. Pennock, and L.Q.M. Chow, "The evolving role of immune checkpoint inhibitors in cancer treatment," The Oncologist, vol. 20, no. $7, \quad$ pp. 812-822, July 2015, https://doi.org/10.1634/theoncologist.2014-0422.

[78] A. Haslam, and V. Prasad, "Estimation of the percentage of US patients with cancer who are eligible for and respond to checkpoint 
inhibitor immunotherapy drugs," JAMA Network Open, vol. 2, no. 5, pp. 1-9, May 2019, https://doi.org/10.1001/jamanetworkopen.2019.2535.

[79] D. Schadendorf, F.S. Hodi, C. Robert, J.S. Weber, K. Margolin, O. Hamid, D. Patt, T.T. Chen, D.M. Berman, and J.D. Wolchok, "Pooled analysis of long-term survival data from phase II and phase III trials of ipilimumab in unresectable or metastatic melanoma," Journal of Clinical Oncology, vol. 33, no. 17, pp. 1889-94, June 2015, https://doi.org/10.1200/JCO.2014.56.2736.

[80] C. Robert, R. Ribas, O. Hamid, A. Daud, J.D. Wolchok, A.M. Joshua, W-J. Hwu, J.S. Weber, T.C. Gangadhar, R.W. Joseph, R. Dronca, A. Patnaik, H. Zarour, R. Kefford, P. Hersey, J. Zhang, J. Anderson, S.J. Diede, S. Ebbinghaus. and F.S. Hodi, "Durable complete response after discontinuation of pembrolizumab in patients With metastatic melanoma," Journal of Clinical Oncology, vol. 36, no. 17, pp. 16681674, June 2018, https://doi.org/10.1200/JCO.2017.75.6270.

[81] S.L. Topalian, F.S. Hodi, J.R. Brahmer, S.N. Gettinger, D.C. Smith, D.F. McDermott, J.D. Powderly, J.A. Sosman, M.B. Atkins, P.D Leming, D.R.Spigel, S.J. Antonia, A. Drilon, J.D. Wolchok, R.D. Carvajal, M.B. McHenry, F. Hosein, C.T. Harbison, J.F. Gross, and M. Sznol, "Five-Year survival and correlates among patients with advanced melanoma, renal cell carcinoma, or non-small cell lung cancer treated with nivolumab," JAMA Oncology, vol. 5, no. 10, pp. 1411-1420, July 2019, https://doi.org/10.1001/jamaoncol.2019.2187.

[82] M.S. Goldberg, "Immunoengineering: how nanotechnology can enhance cancer immunotherapy," Cell, vol. 161, pp. 201-204, April 2015, https://doi.org/10.1016/j.cell.2015.03.037.

[83] Y. Min, K.C. Roche, and S. Tian, "Antigen-capturing nanoparticles improve the abscopal effect and cancer immunotherapy," Nature Nanotechnology, vol. 12, no. 9, 877-882, June 2017, https://doi.org/10.1038/nnano.2017.113.

[84] F.F. Gellrich, M. Schmitz, S. Beissert, and F. Meier, "Anti-PD-1 and novel combinations in the treatment of melanoma - an update," Journal of Clinical Medicine, vol. 9, no. 1, p. 223, January 2020, https://doi.org/10.3390/jcm9010223.

[85] I. Morales-Orue, R. Chicas-Sett, and P.C. Lara, "Nanoparticles as a promising method to enhance the abscopal effect in the era of new targeted therapies," Reports of Practical Oncology and Radiotherapy, vol. 24, no. 1, pp. 86-91, January 2019, https://doi.org/10.1016/j.rpor.2018.11.001.

[86] G. Köhler and C. Milstein "Continuous cultures of fused cells secreting antibody of predefined specificity," Nature, vol. 256, no. 5517, pp. 495-497, September 1975 https://doi.org/10.13140/RG.2.2.33835.90401

[87] J. Lohmueller, and O.J. Finn, "Current modalities in cancer immunotherapy: immunomodulatory antibodies, CARs and vaccines," Pharmacology and Therapeutics, vol. 178, pp. 31-47, March 2017, https://doi.org/10.1016/j.pharmthera.2017.03.008.

[88] A.M. Scott, J.D. Wolchok, and L.J. Old, "Antibody therapy of cancer," Nature Reviews Cancer, vol. 12, no. 4, pp. 278-287, March 2012, https://doi.org/10.1038/nrc3236.

[89] P.A. Ascierto, and F.M. Marincola, "2015: The year of anti-PD-1/PDL1s against melanoma and beyond," EBioMedicine, vol. 2, no. 2, pp. 92-93, February 2015, https://doi.org/10.1016/j.ebiom.2015.01.011.

[90] D. Zahavi, and L. Weiner, "Monoclonal antibodies in cancer therapy," Antibodies, vol. 9, no. 3, p. 34, July 2020 , https://doi.org/10.3390/antib9030034.

[91] M.S. Castelli, P. McGonigle, and P.J. Hornby "The pharmacology and therapeutic applications of monoclonal antibodies," Pharmacology Research and Perspectives, vol. 7, no. 6, pp. 1-11, December 2019, https://doi.org/10.1002/prp2.535.

[92] V. Janelle, C. Rulleau, S.D. Testa, C. Carli, and J-S. Delisle, "T-cell immunotherapies targeting histocompatibility and tumor antigens in hematological malignancies," Frontiers in Immunology, vol. 11, no. 276, pp. 1-15, February 2020, https://doi.org/10.3389/fimmu.2020.00276.

[93] A. Karlitepe, O. Ozalp, and C.B. Avci, "New approaches for cancer immunotherapy," Tumor Biology, vol. 36, no. 6, pp. 4075-4078, May 2015, https://doi.org/10.1007/s13277-015-3491-2.

[94] M. Sambi, L.Bagheri, and M.R. Szewczuk, "Current challenges in cancer immunotherapy: multimodal approaches to improve efficacy and patient response rates," Journal of Oncology, vol. 2019, no. 508794, $\quad$ pp. 1-12, $\quad$ February 2019 , https://doi.org/10.1155/2019/4508794.

[95] I. Melero, G. Gaudernack, W. Gerritsen, C. Huber, G. Parmiani, S. Scholl, N. Thatcher, J. Wagstaff, C. Zielinski, I. Faulkner, and H. Mellstedt, "Therapeutic vaccines for cancer: an overview of clinical trials," Nature Reviews Clinical Oncology, vol. 11, no. 9, pp. 509524, September 2014, https://doi.org/10.1038/nrclinonc.2014.111.

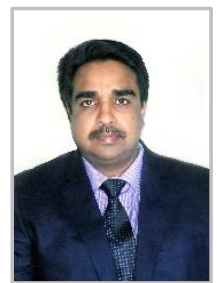

Navas A. A. was born in India. His qualifications include an MD in internal medicine from the University of Mangalore, India (1991), and MBBS - Bachelor of Medicine, Bachelor of Surgery - from the University of Mysore, India (1986).

$\mathrm{He}$ was a consultant physician and research coordinator at the Centre for Research and Fetal Therapy (CRAFT) in Kodungallur, Kerala, India, from 1992-2002. He then pursued his interest in teaching, alongside full time clinical work from 2002-2008 at MES Medical College, Kerala, India, where he was an Assistant Professor of Internal Medicine. In 2008, he joined the Ministry of Health, Brunei, as a physician with academic attributes providing outpatient, inpatient, emergency and intensive care, and significant undergraduate medical teaching. He was in charge of Brunei's National Isolation Centre and contributed to the effective delivery of clinical services in cardiology, diabetes, and endocrinology, and in anticoagulation therapies. $\mathrm{He}$ is currently an Assistant Professor in Internal Medicine at the College of Medicine and Health Sciences in the National University of Science and Technology in Oman. His publications include the articles 'Novel Trends in the Management of Alzheimer's Disease by Using Nano Based Materials' (Madridge Journal of Nanotechnology and Nanoscience, 2018), 'Biomedical Application of Polymers - An Overview' (Current Trends in Biomedical Engineering \& Biosciences, 2018), and 'Versatility of Nanoparticles in Modern Medicine' (Global Journal of Nanomedicine, 2018). His research interests span nanomedicine, cancer nanomedicine, Alzheimer's disease, and cardiac arrhythmias.

Dr. Navas has an enduring interest in the clinical applications of research, especially emerging therapeutic strategies that have the potential to enhance compassionate care and assist patients further, on their journey from complex illness to health.

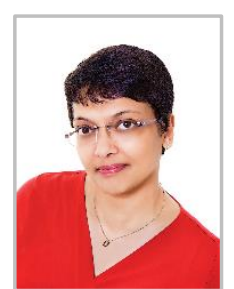

Nandini Doreswamy was born in India. She immigrated to New Zealand in 1994, and to Australia in 2016. Her educational qualifications include a Master of Business Administration (MBA, with distinction, 2019) specialising in managing and leading people, from Southern Cross University, Australia, Master of Surgery in general surgery (MS, 1993) from the University of Mangalore, India, and MBBS Bachelor of Medicine, Bachelor of Surgery - from the University of Mysore, India (1987).

She started her career in medicine in 1987 , specialising as a general surgeon from 1991-94. She was the Surgical Research Officer at the Research Centre for Developmental Medicine and Biology in the University of Auckland, New Zealand, from 1995-1997. She then pursued her interests in technology and management, building a successful career in these fields. In 2002, she co-founded Radius Networks, an award-winning IT company in New Zealand, where she as a Director from 2002-16. From 2016-2017, she worked at the Director level in the Australian federal Department of Health, where her domain skills in health, technology, and management were brought to bear. She currently works in Canberra, Australia, as the Chief Operating Officer of Kokotala, a social enterprise headquartered in Auckland, New Zealand. Her research interests lie at the intersection of disciplines and include health technology, nanomedicine, data science, artificial intelligence, cybersecurity, and healthcare leadership.

Dr. Doreswamy is a Fellow of the Institute of Managers and Leaders, Australia and New Zealand. She is a Member of the National Coalition of Independent Scholars, a Member of the Australian Institute of Company Directors, and a Member of ISACA (Information Systems Audit and Control Association). She was formally recognised by the Australian federal Department of Health as a Distinguished Talent in 2016 and granted Australian Permanent Residence as a Distinguished Talent in 2018.

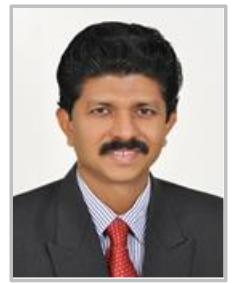

P. J. Joseph Francis was born in India. He has a Master of Science in biochemistry (MSc, 1987), a PhD in nanochemistry (1999), a Master of Computer Applications (MCA, 2001), and a Master of Technology in polymer technology (MTech, 2004) from the Cochin University of Science and Technology, Kochi, India. His Bachelor of Science (BSc, 1984) was in biochemistry, with minors in physics and mathematics, from the University of Kerala, India.

He started his career as Senior Lecturer in polymer chemistry at the Cochin University of Science and Technology (1999-2005). From 20052006, he was Assistant Professor, Applied Chemistry, at Jeppiaar Engineering College in Chennai, India. From August 2006 to January 2012, he was a Senior Lecturer in Applied Chemistry at the Higher College of 
Technology in Muscat, Oman. After a stint as Assistant Professor, Chemistry, at the University of Calicut in India, he returned to Oman in September 2012 to join the College of Medicine and Health Sciences, National University of Science and Technology, as Assistant Professor in Biochemistry. He continues to work in this position today. He has published two books for the Bachelor of Technology program at Anna University, Chennai, India. He has published 25 scientific papers, the most recent being 'Novel Trends in the Management of Alzheimer's Disease by Using Nano Based Materials' (Madridge Journal of Nanotechnology and Nanoscience, 2018), 'Biomedical Application of Polymers - An Overview' (Current Trends in Biomedical Engineering \& Biosciences, 2018), and 'An Overview on Nanotoxicity' (Advances in Nanomedicine and Nanotechnology Research, 2019). His research interests are medicinal chemistry, biochemistry, nanochemistry, phytochemistry, polymer chemistry and technology, environmental chemistry, polymer nanocomposites, and applied chemistry.

Dr Francis was invited by the Government of The Netherlands in 2003, to visit various universities in The Netherlands as part of an academic cooperation initiative with the Cochin University of Science and Technology. He is a consultant on science to international educational and industry organizations and has visited universities, academic and research institutions, and industrial concerns in France, UAE, Oman and India. He is a life member of professional bodies recognised internationally (Asian Polymer Association and the Society for Polymer Technologists) as well as professional associations in India. He is an editorial board member (EBM) and reviewer of the Journal of Nanotechnology in Diagnosis and Treatment, the Global Journal of Nanomedicine, and the International Journal of Nanoparticles and Molecular Nanotechnology. $\mathrm{He}$ is a recognized TRC (The Research Council) Reviewer in Oman. 